\title{
Extracerebral absorption of near infrared light influences the detection of increased cerebral oxygenation monitored by near infrared spectroscopy
}

\author{
Timothy J Germon, Amber E R Young, Alexander R Manara, Richard J Nelson
}

\begin{abstract}
The detection of increased cerebral oxygenation secondary to cerebral hyperaemia, induced by hypercapnia has been studied in anaesthetised patients using a near infrared, reflectance mode, cerebral oxygenation monitor (Invos 3100 Somanetics, Troy, Michigan, USA). Two studies were performed, with and without a pneumatic scalp tourniquet, to distinguish between extracranial and intracranial changes in tissue oxygenation. In the control study a mean increase in end tidal $\mathrm{CO}_{2}$ of $23.1 \mathrm{~mm} \mathrm{Hg}$ was accompanied by a mean increase in middle cerebral artery flow velocity of $116 \%$. Regional cerebral oxygen saturation $\left(\mathrm{rSo}_{2}\right)$ measured transcutaneously in the frontal distribution of the middle cerebral artery increased significantly from $70.5 \%$ to $74 \cdot 6 \%(p=0.001)$. During the second study with a scalp tourniquet inflated to maintain the extracranial tissues in a state of stable ischaemia a mean increase in end tidal $\mathrm{CO}_{2}$ of $22.3 \mathrm{~mm} \mathrm{Hg}$ was accompanied by a mean increase in middle cerebral artery flow velocity of $121 \%$. The change in $\mathrm{rSo}_{2}$ from $62.6 \%$ to $64.5 \%$ was not significant $(p=0.085)$. There was no correlation between the change in middle cerebral artery flow velocity and $\mathrm{rSO}_{2}$ in the control or scalp ischaemia group.
\end{abstract}

This study shows that the Invos 3100 monitor is sensitive to tissue oxygenation but does not reliably detect changes in cerebral oxygenation as a result of profound cerebral hyperaemia. The contribution of extracerebral tissue to the attenuation of near infrared light and the lack of spatial resolution remain major problems to be overcome before this or other near infrared spectroscopy instruments can be introduced into clinical practice.

(F Neurol Neurosurg Psychiatry 1995;58:477-479)

Keywords: near infrared spectroscopy; increased cerebral oxygenation

Near infrared spectroscopy, a non-invasive technique for monitoring cerebral oxygena- tion and blood flow, semiquantitatively estimates the relative concentrations of oxyhaemoglobin and deoxyhaemoglobin within an unspecified volume of tissue illuminated by near infrared light of varying wavelengths. Absorption of the near infrared light takes place in the arterial, capillary, and venous circulations of both the extracranial and intracranial tissues. The technique possesses no intrinsic compartmental or spatial resolution and thus to be clinically useful the effects of changes in extracranial blood flow and oxygenation must be either quantified or made negligible by the design of the optical sensor and its software.

We have examined the capacity of the Invos 3100 cerebral oximeter to detect profound increases in cerebral blood flow and oxygenation induced by hypercapnia before and after the application of a pneumatic scalp tourniquet which results in stable scalp ischaemia.

\section{Methods}

Eight patients (mean age $44 \cdot 5$, range $30-66$ years) undergoing cervical or lumbar discectomy with no predisposition to cerebrovascular disease gave informed verbal consent to the study, which was approved by the local ethics committee. A uniform anaesthetic technique was employed. The electrocardiogram (Hewlett Packard 78353B, GMBH, Germany), arterial blood pressure (Dinamap $845 \mathrm{XT}$, Critikon, Tampa, FL, USA), regional cerebral $\mathrm{O}_{2}$ saturation $\left(\mathrm{rSO}_{2}\right)$, inspired $\mathrm{O}_{2}$ and $\mathrm{CO}_{2}$ concentrations, and end tidal $\mathrm{CO}_{2}\left(\mathrm{ETCO}_{2}\right)$ concentrations (Nellcor N-1000, Hayward, CA, USA) were continuously monitored. The oximeter sensor was placed on the forehead, avoiding the midline and the temporalis muscle. Ipsilateral middle cerebral artery flow velocity was measured in the proximal middle cerebral artery at a depth of 5.0 to $6.0 \mathrm{~cm}$ with transcranial Doppler ultrasonography (PCDop 842, Scimed, Bristol, UK).

When $\mathrm{ETCO}_{2}$ was stable in the range $22-45 \mathrm{~mm} \mathrm{Hg}$ five recordings were made. The inspired $\mathrm{CO}_{2}$ concentration was increased to $5 \%$ and when $\mathrm{ETCO}_{2}$ had reached a stable level for three minutes five further recordings were made. The study was then repeated with a pneumatic tourniquet 
Mean values (SD) for the variables measured during normocapnia and hypercapnia in control and scalp ischaemia studies

\begin{tabular}{|c|c|c|c|c|c|c|}
\hline & \multicolumn{3}{|l|}{ Control study } & \multicolumn{3}{|c|}{ Scalp ischaemia } \\
\hline & Normocapnia & Hypercapnia & Pvalue & Normocapnia & Hypercapnia & Pvalue \\
\hline $\begin{array}{l}\text { Heart rate }(6 \mathrm{pm}) \\
\mathrm{MAP}(\mathrm{mm} \mathrm{Hg}) \\
\mathrm{ETCO}_{2}(\mathrm{~mm} \mathrm{Hg}) \\
\mathrm{vMCA}(\mathrm{cm} / \mathrm{s}) \\
\mathrm{rSO}_{2}(\%)\end{array}$ & $\begin{array}{l}66(10) \\
77(7) \\
28 \cdot 6(6) \\
37(13) \\
70 \cdot 5(4)\end{array}$ & $\begin{array}{l}63(9) \\
83(8) \\
51 \cdot 7(5) \\
80(23) \\
74 \cdot 6(4)\end{array}$ & $\begin{aligned} & 0.05 \\
& 0.05 \\
&<0.01 \\
&< 0.01 \\
& 0.001\end{aligned}$ & $\begin{array}{l}64(13) \\
79(7) \\
28 \cdot 9(6) \\
38(17) \\
62 \cdot 6(8)\end{array}$ & $\begin{array}{l}63(12) \\
82(10) \\
51 \cdot 2(6) \\
84(24) \\
64 \cdot 5(10)\end{array}$ & $\begin{array}{l}0.44 \\
0.03 \\
<0.01 \\
<0.01 \\
0.085\end{array}$ \\
\hline
\end{tabular}

inflated to a pressure of $200 \mathrm{~mm} \mathrm{Hg}$ at the level of the supraorbital ridge for three minutes. Care was taken to ensure that the tourniquet did not impinge on the sensor.

A paired Student's $t$ test was used to examine for significant differences between the study variables under normocapnic and hypercapnic conditions. The relation between the percentage change in middle cerebral artery velocity and the change in $\mathrm{rSO}_{2}$ was studied with Pearson's rank correlation.

\section{Results}

The table summarises the results. The mean increase in $\mathrm{ETCO}_{2}$ was 23.1 (SD 4) $\mathrm{mm} \mathrm{Hg}$ in the control study and 22.3 (3) $\mathrm{mm} \mathrm{Hg}$ when the scalp tourniquet was inflated. (In one patient without a satisfactory acoustic window for transcranial Doppler, $\mathrm{ETCO}_{2}$ rose by $20 \mathrm{~mm} \mathrm{Hg}$ without the tourniquet and by $23 \mathrm{~mm} \mathrm{Hg}$ with the tourniquet, and therefore, his $\mathrm{rSO}_{2}$ results are included.) There was no significant difference between the mean increase in middle cerebral artery velocity in the two studies $(p=0 \cdot 21)$. There was a significant increase in mean $\mathrm{rSO}_{2}$ from $70.5 \%$ to $74.6 \%$ in the control study $(p=0.001$, figure). Under conditions of stable extracranial ischaemia, however, no significant change in $\mathrm{rSO}_{2}$ was detected in response to hypercapnia. (Mean $\mathrm{rSO}_{2}$ increased from $62.6 \%$ to $64.5 \%$, $\mathrm{p}=0.085$, figure). There was no correlation between the changes in middle cerebral artery velocity and $\mathrm{rSO}_{2}$ in the control $(\mathrm{r}=0.229$, $\mathrm{p}=0.621)$ or scalp ischaemia $(\mathrm{r}=0.183$, $\mathrm{p}=0.398$ ) groups.

\section{Discussion}

Carbon dioxide is a potent cerebral vasodilator. The increase in cerebral blood flow mediated by cortical arteriolar dilatation ${ }^{1}$ in response to increased $\mathrm{CO}_{2}$ does not increase cerebral metabolic requirements for oxygen. ${ }^{2}$ This results in an increased cerebral blood volume $^{3}$ of oxygenated arteriolar blood and an increase in the saturation of cerebral venous blood secondary to increased blood flow with constant $\mathrm{O}_{2}$ extraction. It follows that $\mathrm{rSO}_{2}$ should increase significantly in response to hypercapnia and there should be a close relation between increased flow velocity detected by transcranial Doppler and increased cerebral oxygenation detected by near infrared spectroscopy.

Assuming that $70 \%$ of cerebral blood volume is venous and $30 \%$ arterial and that normal jugular venous oxygen $\left(\mathrm{SjO}_{2}\right)$ saturation lies in the range $54 \%$ to $75 \%$ then the normal range for $\mathrm{rSO}_{2}$ should lie between $67 \%$ and $85 \%$. Mean $\mathrm{rSO}_{2}$ in our control study was $70.5 \%$. The increase in middle cerebral artery velocity in the two studies is compatible with an increase in global cerebral blood flow of about $120 \%$. Assuming no appreciable change in the distribution of cerebral blood volumes or the oxygen dissociation curve for haemoglobin and a mean $\mathrm{SjO}_{2}$ in the midrange of normal (65\%) during normocapnia, we estimate that a $120 \%$ increase in cerebral blood flow would increase $\mathrm{rSO}_{2}$ from $76 \%$ to $88 \%$. With the same rationale, the mean normocapnic value for $\mathrm{rSO}_{2}$ of $70.5 \%$ should have increased to $86 \%$. Although the increase in $\mathrm{rSO}_{2}$ from $70.5 \%$ to $74.6 \%$ in the

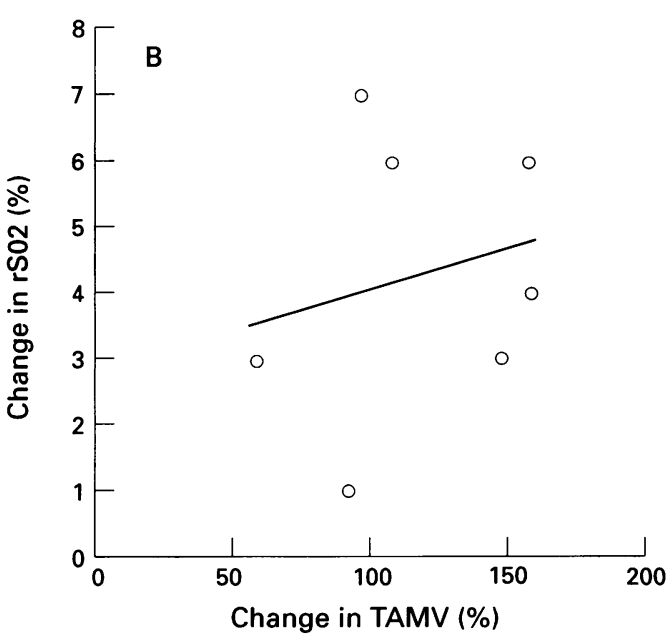

(A) Individual (circles) and mean (stars) values of $r \mathrm{SO}_{2}$ for each of the four study conditions:

$a=$ control, normocapnia;

$b=$ control, hypercapnia;

$c=$ scalp ischaemia ,

normocapnia; $d=$ scalp

ischaemia, hypercapnia.

(B) Scatter plot of change in $r \mathrm{SO}_{2}$ against \% change in time averaged mean

flow velocity (TAMV) in the middle cerebral artery in response to hypercapnia under control conditions (no scalp ischaemia, $r=0.229, p=0.621$ ). 
control study was significant it clearly does not reflect the magnitude of change predicted and further, there was no correlation between changes in middle cerebral artery flow velocity and $\mathrm{rSO}_{2}$ in either study.

In an attempt to overcome the problems of extracerebral attenuation of near infrared light and to increase the degree of spatial resolution the cerebral oximeter used in this study has two receiving optodes placed 3 and $4 \mathrm{~cm}$ from the emitting optode. Subtraction of the absorption characteristics detected at the proximal optode from those at the distal optode should reflect changes taking place in cerebral tissue alone. ${ }^{4}$ With this optode arrangement we have previously shown that the induction of scalp ischaemia causes a significant reduction in $\mathrm{rSO}_{2}$ as measured by the Invos $3100 .^{5}$ This may mean the mathematical algorithm that computes $\mathrm{rSO}_{2}$ may not compensate for the changes in scalp oxygenation. It seems unlikely that the baseline mean $\mathrm{rSO}_{2}$ of $62.6 \%$ in the second study reflects true regional $\mathrm{O}_{2}$ saturation in the stable anaesthetised patient. Nevertheless, the principle of the proximal and distal path lengths should apply to the new stable conditions with the sensor capable of detecting the cerebral hyperaemia unaffected by extracranial change. The small and statistically insignificant increase in $\mathrm{rSO}_{2}$ to $64.5 \%$ in the presence of scalp ischaemia strongly suggests that the increase in $\mathrm{rSO}_{2}$ seen in response to hypercapnia in the absence of the tourniquet is largely due to changes in scalp oxygenation.

With this optode arrangement, the Invos 3100 monitor will detect tissue hypoxia deep to the scalp under conditions identical with those described. ${ }^{5}$ We speculated whether the monitor was detecting changes in haemoglobin oxygenation in the diploic frontal bone and underlying dura, in the cerebral cortex, or in both. The failure to detect changes in oxygenation in response to hypercapnia with scalp ischaemia suggests that very little of the near infrared light signal change is taking place in the cerebral cortex and that hypercapnia does not increase blood flow to the skull. In similar work Harris and Bailey failed to detect any significant response to hypercapnia using a sensor configuration with a smaller separation between the light source and the two receivers. ${ }^{6}$ It is possible that this sensor geometry was successful in excluding hyperaemic change in the scalp while remaining insensitive to change in the cerebral substance.

The problem of extracerebral attenuation of near infrared light in near infrared spectroscopy is not simply a theoretical consideration. Those patients most likely to benefit from this monitoring are also those most likely to have local change in the extracranial tissues-for example, after head injury or craniotomy-and systemic changes in cardiac output, peripheral resistance, and cutaneous vasodilatation, which also affect the extracranial tissues. Until the problems of quantifying and interpreting changes in $\mathrm{rSO}_{2}$ under physiological and pathological conditions have been overcome near infrared spectroscopy monitors of this sort cannot be recommended for routine clinical monitoring. Other instruments with similar technology should be tested equally rigorously.

This work was supported by the Stroke Association. We also gratefully acknowledge the assistance of the Departments of Medical Physics and Medical Engineering, Frenchay Hospital, the financial support of Frenchay WRVS, and the technical assistance of Neurotechnics Ltd, Thame, Oxon.

This work was presented in part at the inaugural meeting of the British Neurosurgical Research Group, Newcastle-uponTyne, April 1994.

1 Symon L. Pathological regulation in cerebral ischaemia. In: Wood JH, ed. Cerebral blood flow, physiologic and clinical aspects. New York: McGraw-Hill, 1987:413-24.

2 Kety SS, Schmidt CF. The effects of altered arterial tensions of carbon dioxide and oxygen on cerebral blood flow and cerebral oxygen consumption of normal young men. $\mathcal{F}$ Clin Invest 1948;27:484.

3 Greenberg JH, Alavi A, Reivich M, Kuhl D, Uzzell B. Local cerebral blood volume response to carbon dioxide in man. Circ Res 1978;43:324-31.

4 McCormick PW, Stewart M, Goetting MG, Balakrishnan $G$. Regional cerebrovascular oxygen saturation measured by optical spectroscopy in humans. Stroke 1991; 22:596-602

5 Germon TJ, Kane NM, Manara AR, Nelson RJ. Near infrared spectroscopy in adults: the effects of extracranial ischaemia and intracranial hypoxia on the estimation of cerebral oxygenation. Br f Anaesth 1994;43. 503-6.

6 Harris DNF, Bailey SM. Near infrared spectroscopy in adults. Anaesthesia 1993;48:694-6. 